\title{
Serum Cytokeratin-18 Levels for Liver Fibrosis Prediction
}

\author{
Gian Paolo Caviglia ${ }^{1, *}$; Rinaldo Pellicano ${ }^{2}$ \\ ${ }^{1}$ Department of Medical Sciences, University of Turin, Turin, Italy \\ ${ }^{2}$ Department of Gastroenterology, Citta della Salute e della Scienza, Molinette Hospital, Turin, Italy \\ ${ }^{*}$ Corresponding Author: Gian Paolo Caviglia, Department of Medical Sciences, University of Turin, Turin, Italy. Tel: +39-0116333922, Fax: +39-0116333976, E-mail: caviglia.giampi@libero.it
}

Received: June 17, 2015; Accepted: July 4, 2015

Keywords: Cytokeratin-18; Liver Fibrosis; Hepatitis

\section{Dear Editor,}

We have read with great interest the meta-analysis by Yang et al. (1). The reported objective was to evaluate the relation between cytokeratin-18 (CK-18) levels and hepatitis pathogenesis. Eight case-control studies were included in the meta-analysis according to the selection criteria adopted. High serum CK-18 levels were found in patients with hepatitis compared to controls. Moreover, increased serum CK-18 levels were related to fibrosis progression irrespective of ethnicity (Asians, Africans, and Caucasians) and liver disease etiology (chronic hepatitis $\mathrm{C}$, chronic hepatitis $\mathrm{B}$, and nonalcoholic steatohepatitis [NASH]). The authors concluded that serum CK-18 could be adopted as a diagnostic marker of liver injury and as a predictor of hepatitis progression (1).

Although the authors correctly reported the presence of several specific limitations, it should be highlighted that the pathogenetic mechanism leading to liver damage and fibrogenesis varies according to the underlying liver disease etiology. CK-18 is a serological marker of apoptosis. Although circulating levels of CK-18 have been shown to be elevated in different liver disorders $(2,3)$, hepatocyte apoptosis seems to play a critical role in liver injury only in the setting of nonalcoholic fatty liver disease (NAFLD) to NASH evolution (4). Moreover, the clinical value of CK-18 for NASH diagnosis and fibrosis severity prediction has been recently questioned in a study examining CK-18 relationship with clinical/metabolic and histologic parameters in a large multiethnic NAFLD population. In this study, not included in the meta-analysis, CK-18 showed just a modest correlation with the severity of fibrosis $(r=0.32)$ and lobular inflammation $(r=0.28)$. In addition, the performance for NASH and fibrosis diagnosis was poor as reflected by values of area under the curve (AUC) of 0.65 , sensitivity (Se) of 58\%, and negative predictive value (NPV) of $49 \%$ and AUC of 0.68 , Se of $54 \%$, and NPV of $56 \%$, respectively (5).

In conclusion, CK-18 diagnostic value in distinguishing NAFLD from NASH has been currently reconsidered. Further studies are required to explore the possible use of CK-18 in combination with other noninvasive markers of fibrosis to achieve a more accurate diagnosis of NASH and fibrosis severity.

\section{Authors' Contributions}

Gian Paolo Caviglia wrote the manuscript. Rinaldo Pellicano revised it critically.

\section{References}

1. Yang ZH, Yang SX, Qin CZ, Chen YX. Clinical values of elevated serum cytokeratin-18 levels in hepatitis: a meta-analysis. Hepat Mon. 2015;15(5):e25328.

2. Caviglia GP, Ciancio A, Rosso C, Abate ML, Olivero A, Pellicano R, et al. Non-invasive methods for the assessment of hepatic fibrosis: transient elastography, hyaluronic acid, 13C-aminopyrine breath test and cytokeratin 18 fragment. Ann Hepatol. 2013;13(1):91-7.

3. Papatheodoridis GV, Hadziyannis E, Tsochatzis E, Chrysanthos N, Georgiou A, Kafiri G, et al. Serum apoptotic caspase activity as a marker of severity in HBeAg-negative chronic hepatitis B virus infection. Gut. 2008;57(4):500-6.

4. Eguchi A, Wree A, Feldstein AE. Biomarkers of liver cell death. J Hepatol. 2014;60(5):1063-74.

5. Cusi K, Chang Z, Harrison S, Lomonaco R, Bril F, Orsak B, et al. Limited value of plasma cytokeratin-18 as a biomarker for NASH and fibrosis in patients with non-alcoholic fatty liver disease. $J$ Hepatol. 2014;60(1):167-74. 